\title{
Hierarchy-Enhancing Misinformation: \\ Social Dominance Motives Are Uniquely Associated With Republicans' Belief In and Sharing of Election-Related Misinformation
}

\author{
Jeffrey Lees ${ }^{1 *}$, Victoria A. Parker ${ }^{2}$
}

This manuscript has not undergone peer-review

${ }^{1}$ Media Forensics Hub, Clemson University

${ }^{2}$ Department of Psychology, Wilfrid Laurier University

* Corresponding Author: Jeffrey Lees (jeffrey.m.lees@gmail.com)

Open Materials: https://osf.io/tpc6w/

Keyworks: Misinformation, social dominance, 2020 US election, Donald Trump 


\begin{abstract}
The aftermath of the 2020 US Presidential election saw a deluge of election-related misinformation which falsely asserted that the election was "stolen" from Donald Trump. Since then a majority of Republicans have consistently expressed belief in this misinformation, despite no evidence for its veracity and its motivating role in the January 6th, 2021 attack on the US Capitol. Here we present evidence, using a repeated-measures design $(N=355)$ across a highly generalizable stimulus set, that Republicans' support for 2020 US election-related misinformation and willingness to share it on social media are uniquely associated with social dominance motives, along with conspiracy mentality and party identification strength. We find little evidence that right-wing authoritarianism is associated with the belief in or sharing of election-related misinformation, and that cognitive reflectiveness is only associated with sharing, but not belief. We introduce the theoretical lens of Hierarchy-Enhancing Misinformation to interpret these findings, arguing that election-related misinformation is best understood as a functional mechanism by which group-based dominance hierarchies are socially and psychologically reinforced.
\end{abstract}




\section{HIERARCHY-ENHANCING MISINFO}

\section{Introduction}

Misinformation about the validity of elections has the power to distort normal political processes and erode democratic values. The aftermath of the 2020 US Presidential election was a stark illustration of the dangers of election-related misinformation and conspiracy theories online. Despite the motivating role this misinformation played in the January 6th attack on the US Capitol Complex which left five dead, almost two thirds of the House Republican caucus voted to reject the election results. And on November 10th, 2020, only seven days after the 2020 US election, $65 \%$ of Republicans believed Trump was more likely the winner than Biden (Pennycook \& Rand, 2021b). By August of 2021, 78\% of Republicans claimed they do not believe Biden legitimately won enough votes to be President, compared to $3 \%$ of Democrats and $35 \%$ of Independents (Agiesta \& Edwards-Levy, 2021).

Researchers have recently suggested that a primary contributor to belief in and sharing of misinformation is a lack of analytical thinking, where a lack of cognitive effort and attentiveness, not partisan motivated reasoning, best explains the proliferation of misinformation on social media (Pennycook \& Rand, 2021a, 2019; Ross et al., 2021). A lack of attentiveness has also been implicated in the disconnect between what individuals believe and what they choose to share over social media, as people will indicate a willingness to share an article which, if asked, they would label as containing misleading information (Pennycook, Epstein, et al., 2021). Other work has highlighted the potential benefits of fact-checking misinformation (Nyhan et al., 2020; Walter et al., 2020) and "inoculation" against it by priming individuals to expect to be deceived prior to exposure (Maertens, Roozenbeek, et al., 2020; Roozenbeek \& van der Linden, 2019).

Nonetheless, much of this work focuses on genericized political misinformation, for example a headline falsely claiming Hillary Clinton was intoxicated the night of the 2016 US election (though note work on reducing climate change denialism, e.g., Maertens et al., 2020). As such, work on domain-general mechanisms of misinformation belief may not generalize well to specific misinformation contexts, such as election-related misinformation or misinformation explicitly endorsed by conservative elites, which may better activate ideological mechanisms of belief including conservatives' lower sensitivity to falsehoods (Garrett \& Bond, 2021), greater preference for homogenous social networks and conformity (Jost et al., 2018), or authoritarian motives in support for Donald Trump (Choma \& Hanoch, 2017; Womick et al., 2019).

Here we introduce the concept of "Hierarchy Enhancing Misinformation" to describe misinformation whose primary purpose is to enforce existing social hierarchies, and leverage this concept to understand Republicans' support for misinformation which challenges the validity of the 2020 US Presidential election.

The preference for group-based hierarchies and inequality can be understood as an individual trait called Social Dominance Orientation (SDO) (Ho et al., 2015; Sidanius et al., 2016), and is a notable predictor of intergroup prejudice (Bratt et al., 2016; Ho et al., 2012), selfishness (Halali et al., 2018), unethical behaviors (Son Hing et al., 2007), and less concern about harm (Federico et al., 2013). Individuals high in SDO wish to maintain and enhance social hierarchies through group-based dominance and anti-egalitarian ideologies, while those low in 


\section{HIERARCHY-ENHANCING MISINFO}

SDO seek to attenuate social hierarchies and embrace more egalitarian worldviews. Democratic institutions and processes are inherently hierarchy-attenuating as they provide relatively equal participation in governance regardless of an individual's or group's position in the social hierarchy. More acutely, the 2020 US election saw the loss of Donald Trump, the support for whom is uniquely associated with authoritarian and dominance motives (Choma \& Hanoch, 2017; Womick et al., 2019). This creates an environment where both partisan and hierarchy-enhancing motives are well positioned to reinforce the belief that the 2020 US election was fraudulent.

We propose that the motivation to enhance social hierarchies through group-based dominance is associated with belief in and sharing of election-related misinformation among US Republicans, precisely because fair and free elections in the United States may induce a feeling of threat among consevative voters of the rising political power of non-White Americans (Craig \& Richeson, 2014b, 2014a) which threatens long held myths of racial progress in American political culture (Kraus et al., 2017; Onyeador et al., 2021). The current study aims to establish SDO's unique association with the belief in and sharing of election-related misinformation among Republicans, in comparison to alternative psychological correlates including cognitive reflectiveness, right-wing authoritarianism, general conspiracy mentality, and party identification strength across a generalizable set of 2020 US election-related misinformation.

\section{Method}

\section{Open Science}

Study design, measures, sample size, hypotheses, and analyses were all preregistered (https://osf.io/jd72f). All measures, stimuli, data, and analyzes presented herein and required for replication are publicly available (https://osf.io/tpc6w/).

\section{Participants}

We recruited 381 participants on the Prolific survey platform (Palan \& Schitter, 2018) who had previously identified as Republicans in Prolific's prescreen survey. Data were collected in June 2021. Participants were recruited for a 15 minute study and paid \$3.00 USD. 13 participants $(3.41 \%)$ failed the attention check and were excluded from all analyses. 11 participants (2.99\%) identified as Democrats, and 13 (3.53\%) as Independents (1 Leaning Democrat, 12 Leaning Republican), in our survey. The Democrats and Democrat-leaning Independents were excluded from all analyses. The 12 Republican-leaning Independents were included (per the preregistration), leaving a final $N=355$ (53.8\% Male, 46\% Female, 60.9\% with a 4-year college degree or higher, 75\% White, 12.8\% Black, 7.9\% Latinx, 3.8\% Asian, 0.5\% Mixed/unlisted, $M_{\text {age }}=38.03, S D_{\text {age }}=12.35$ )

\section{Measures \& Procedure}

All participants provided informed consent to participate. Participants first indicated their party identification and identification strength, using the measures from the 2016 American 


\section{HIERARCHY-ENHANCING MISINFO}

National Election Survey (ANES; https://electionstudies.org/). Participant then responded to the following four primary independent variables in random order: The 3-item numeric (Frederick, 2005) and 4-item non-numeric (Thomson \& Oppenheimer, 2016) Cognitive Reflection Tasks (CRT), the 16-item Social Dominance Orientation Scale (SDO $;$; Ho et al., 2015), the 18-item short version of the "ACT" Right-Wing Authoritarianism Scale (RWA; Duckitt et al., 2010), and the 5-item Conspiracy Mentality Questionnaire (CMQ; Bruder et al., 2013). Participants then responded to an attention check which asked "Please, in the box below, write out the answer to the following math problem, capitalizing the first letter of your answer (e.g. "Eight", not "eight" or "8"). What does one plus three equal?".

Next, participants viewed a random set of 10 election-misinformation stimuli from a larger set of 50, for example headlines and social media posts. Of the stimuli, 43 contained false information about the validity of the 2020 U.S. Presidential election, and 7 contained true information. All 50 stimuli were the direct subject of a PolitiFact article (https://www.politifact.com/article/2020/nov/20/fact-checking-false-claims-about-2020-election/ ) that either directly refuted the false claim or verified the true claim. All stimuli were intentionally left in the "natural" state they would be in if they appeared on social media, both out of necessity (much of the misinformation had been removed from social media sites and only exists on internet archives), and out of theoretical concern with verisimilitude and external validity. All stimuli, along with documentation of the fact checking for each stimuli, can be found on the OSF (https://osf.io/tpc6w/). For each stimuli participants were asked how likely they would be to share it (1-6 Likert, "Extremely Unlikely" to "Extremely Likely"), how accurate they believed the information in the stimuli was (1-6 Likert; "Extremely Inaccurate" to "Extremely Accurate), both of which were adapted from Pennycook et al. (2021). Participants were also asked to respond to a measure of perceived threat, "Assuming the above post is entirely accurate, how threatening to the American way of life would the information be?" (1-6 Likert, "Not at all Threatening" to "Extremely Threatening"). Item order was fully randomized.

Participants were then asked for basic demographic information, given the opportunity to provide an open comment, then were debriefed. In the debrief they were directly informed that most of the information they viewed was objectively false, according to reputable Fact Checkers, and provided a link to the www.PolitiFact.com article where they could find the specific refutation of every piece of stimuli in the study. Participants then had to view the seven headlines which were verified as true, and study then ended.

\section{Results}

See Table 1 for descriptive statistics and correlation for all individual difference measures. All primary analyses were conducted using linear mixed-effects modeling via the lme4 package v. 1.1-27.1 (Bates et al., 2015) in R v. 4.1.1. All models utilized REML estimation, and degrees of freedom and $p$-values were computed using Satterthwaite's approximation. All models below are linear mixed-effects models with random intercepts for participant and stimuli, and 
random slopes for all predictors which did not cause model convergence issues, per the preregistered analysis plan (Barr et al., 2013; Judd et al., 2017).

Table 1

\begin{tabular}{|l|l|l|l|l|l|l|l|l|}
\hline & Mean & \multicolumn{1}{|c|}{ SD } & Alpha & Scale & SDO & RWA & CMQ & $\begin{array}{c}\text { GOP ID } \\
\text { Str }\end{array}$ \\
\hline SDO & 3.37 & 1.31 & 0.92 & $1-7$ & & & & \\
\hline RWA & 4.58 & 0.94 & 0.85 & $1-7$ & $0.148^{* *}$ & & & \\
\hline CMQ & 5.15 & 1.26 & 0.85 & $1-7$ & 0.092 & $0.170^{* *}$ & & \\
\hline $\begin{array}{l}\text { GOP ID } \\
\text { Str }\end{array}$ & 3.44 & 1.27 & & $1-5$ & $0.212^{* * *}$ & $0.287^{* * *}$ & $0.240^{* * *}$ & \\
\hline CRT & 3.11 & 1.73 & & $0-7$ & 0.000 & -0.050 & $-0.226^{* * *}$ & $-0.158^{* *}$ \\
\hline
\end{tabular}

Table 1: Pearson correlations with pairwise deletion. ${ }^{*} p<0.05,{ }^{*} p<0.01,{ }^{* * *} p<0.001$

In examining responses to the 43 false stimuli, sharing intentions and belief were positively correlated, controlling for participant and stimuli effects $\left(r_{\text {partial }}=0.52, p<0.001\right)$. $50.4 \%$ of belief responses indicated that the false stimuli were at least "slightly accurate," whereas only $32.5 \%$ of sharing responses indicated they were at least "slightly likely" to share the stimuli on social media $(t(3046)=-10.21, \mathrm{~d}=-0.18, p<0.001)$.

We began the primary analyses by examining the linear relationships between the predictor variables of right-wing authoritarianism (RWA), social dominance orientation (SDO), conspiracy mentality (CMQ), and cognitive reflectiveness (CRT), and belief in election-related misinformation and sharing intentions of election-related misinformation. We then separately entered the strength of identification with the Republican Party (PID Str) as a predictor to examine the robustness of the observed associations. No issues with multicollinearity were observed across all four models in Table 2 (highest VIF $=1.13$ ). As further robustness checks we entered participant demographic variables of age (continuous), gender, ethnicity, education levels as control variables to the models which include party identification strength. For belief in election-related misinformation all observed associations in Table 2 held. For sharing of election-related misinformation the linear association between CRT scores and sharing intentions became insignificant $(B=-0.05, p=0.132)$ upon controlling for demographic variables, while all other observed associations held.

Table 2

\begin{tabular}{|c|c|c|c|c|c|c|c|c|c|c|c|c|}
\hline \multirow[b]{2}{*}{ Predictors } & \multicolumn{3}{|c|}{$\begin{array}{c}\text { Belief in Election } \\
\text { Misinfo }\end{array}$} & \multicolumn{3}{|c|}{$\begin{array}{c}\text { Belief in Election } \\
\text { Misinfo }\end{array}$} & \multicolumn{3}{|c|}{$\begin{array}{c}\text { Sharing Election } \\
\text { Misinfo }\end{array}$} & \multicolumn{3}{|c|}{$\begin{array}{c}\text { Sharing Election } \\
\text { Misinfo }\end{array}$} \\
\hline & $\begin{array}{l}\text { Std. } \\
\text { Beta }\end{array}$ & $95 \% C I$ & $p$ & $\begin{array}{l}\text { Std. } \\
\text { Beta }\end{array}$ & $95 \% C I$ & $p$ & $\begin{array}{l}\text { Std. } \\
\text { Beta }\end{array}$ & $95 \% C I$ & $p$ & $\begin{array}{l}\text { Std. } \\
\text { Beta }\end{array}$ & $95 \% C I$ & $p$ \\
\hline
\end{tabular}


HIERARCHY-ENHANCING MISINFO

\begin{tabular}{|c|c|c|c|c|c|c|c|c|c|c|c|c|}
\hline (Intercept) & -0.00 & $\begin{array}{c}-0.09- \\
0.09\end{array}$ & 0.963 & -0.00 & $\begin{array}{c}-0.09- \\
0.09\end{array}$ & 0.992 & -0.00 & $\begin{array}{c}-0.13- \\
0.13\end{array}$ & 0.972 & -0.00 & $\begin{array}{c}-0.13- \\
0.12\end{array}$ & 0.966 \\
\hline RWA & 0.08 & $\begin{array}{c}0.02- \\
0.14\end{array}$ & 0.008 & 0.05 & $\begin{array}{c}-0.01- \\
0.11\end{array}$ & 0.100 & 0.04 & $\begin{array}{c}-0.02- \\
0.10\end{array}$ & 0.196 & 0.00 & $\begin{array}{c}-0.06- \\
0.06\end{array}$ & 0.973 \\
\hline SDO & 0.12 & $\begin{array}{c}0.05- \\
0.18\end{array}$ & $<0.001$ & 0.09 & $\begin{array}{c}0.03- \\
0.16\end{array}$ & 0.003 & 0.09 & $\begin{array}{c}0.03- \\
0.15\end{array}$ & 0.004 & 0.06 & $\begin{array}{c}0.00- \\
0.12\end{array}$ & 0.046 \\
\hline CMQ & 0.23 & $\begin{array}{c}0.17- \\
0.30\end{array}$ & $<0.001$ & 0.21 & $\begin{array}{c}0.15- \\
0.27\end{array}$ & $<0.001$ & 0.17 & $\begin{array}{c}0.11- \\
0.24\end{array}$ & $<0.001$ & 0.14 & $\begin{array}{c}0.08- \\
0.21\end{array}$ & $<0.001$ \\
\hline CRT & -0.01 & $\begin{array}{c}-0.07- \\
0.05\end{array}$ & 0.725 & 0.00 & $\begin{array}{c}-0.05- \\
0.06\end{array}$ & 0.885 & -0.08 & $\begin{array}{c}-0.15- \\
-0.02\end{array}$ & 0.009 & -0.07 & $\begin{array}{c}-0.13- \\
-0.01\end{array}$ & 0.029 \\
\hline PID Str & & & & 0.13 & $\begin{array}{c}0.06- \\
0.20\end{array}$ & $<0.001$ & & & & 0.18 & $\begin{array}{c}0.10- \\
0.25\end{array}$ & $<0.001$ \\
\hline ICC & 0.35 & & & 0.35 & & & 0.48 & & & 0.49 & & \\
\hline $\mathrm{N}$ & $\begin{array}{l}355_{\text {ID }} \\
50_{\text {Stimuli }}\end{array}$ & & & $\begin{array}{l}355_{\text {ID }} \\
50_{\text {Stimuli }}\end{array}$ & & & $\begin{array}{l}355_{\text {ID }} \\
50_{\text {Stimuli }}\end{array}$ & & & $\begin{array}{l}355_{\text {ID }} \\
50 \text { Stimuli }\end{array}$ & & \\
\hline Observations & 3550 & & & 3550 & & & 3550 & & & 3550 & & \\
\hline $\begin{array}{l}\text { Marginal } \mathrm{R}^{2} \\
\text { Conditional } \\
\mathrm{R}^{2}\end{array}$ & / 0.089 / & 0.412 & & $0.103 /$ & 0.414 & & $0.060 /$ & 0.513 & & $0.086 /$ & 0.530 & \\
\hline
\end{tabular}

Table 2: Mixed-effects regression models predicting belief in and sharing of election-related misinformation among Republicans. All predictors and dependent variables are z-scores.

To examine the role perceived threat played in belief in and willingness to share election-related misinformation we interacted perceived threat with SDO and RWA separately in predicting belief and sharing intentions. The interaction between SDO/RWA and threat constituted an interaction between Level 2 (SDO/RWA) and Level 1 (Threat) variables. As such, perceived threat was cluster centered within stimuli, per the preregistration and aligned with best practices regarding cross-level interactions in multilevel models (Enders \& Tofighi, 2007). Table 3 contains the results of these models. We found that threat positively interacted with RWA, but not SDO, in predicting belief in and sharing of election-related misinformation.

Table 3

\begin{tabular}{|c|c|c|c|c|c|c|c|c|c|c|c|c|}
\hline \multirow[b]{2}{*}{ Predictors } & \multicolumn{3}{|c|}{$\begin{array}{c}\text { Belief in Election } \\
\text { Misinfo }\end{array}$} & \multicolumn{3}{|c|}{$\begin{array}{c}\text { Belief in Election } \\
\text { Misinfo }\end{array}$} & \multicolumn{3}{|c|}{$\begin{array}{c}\text { Sharing Election } \\
\text { Misinfo }\end{array}$} & \multicolumn{3}{|c|}{$\begin{array}{c}\text { Sharing Election } \\
\text { Misinfo }\end{array}$} \\
\hline & $\begin{array}{l}\text { Std. } \\
\text { Beta }\end{array}$ & $95 \% C I$ & $p$ & $\begin{array}{l}\text { Std. } \\
\text { Beta }\end{array}$ & $95 \% C I$ & $p$ & $\begin{array}{l}\text { Std. } \\
\text { Beta }\end{array}$ & $95 \% C I$ & $p$ & $\begin{array}{l}\text { Std. } \\
\text { Beta }\end{array}$ & $95 \% C I$ & $p$ \\
\hline (Intercept) & -0.01 & $\begin{array}{c}-0.09- \\
0.08\end{array}$ & 0.883 & -0.01 & $\begin{array}{c}-0.09- \\
0.08\end{array}$ & 0.885 & -0.01 & $\begin{array}{c}-0.13- \\
0.12\end{array}$ & 0.912 & -0.00 & $\begin{array}{c}-0.13- \\
0.12\end{array}$ & 0.945 \\
\hline RWA & 0.09 & $\begin{array}{c}0.03- \\
0.15\end{array}$ & 0.003 & 0.08 & $\begin{array}{c}0.02- \\
0.13\end{array}$ & 0.009 & 0.06 & $\begin{array}{c}0.00- \\
0.12\end{array}$ & 0.047 & 0.05 & $\begin{array}{c}-0.01- \\
0.11\end{array}$ & 0.130 \\
\hline Threat & 0.07 & $\begin{array}{c}0.02- \\
0.12\end{array}$ & 0.004 & 0.07 & $\begin{array}{c}0.02- \\
0.12\end{array}$ & 0.004 & 0.07 & $\begin{array}{c}0.02- \\
0.13\end{array}$ & 0.011 & 0.07 & $\begin{array}{c}0.02- \\
0.13\end{array}$ & 0.010 \\
\hline
\end{tabular}


HIERARCHY-ENHANCING MISINFO

\begin{tabular}{|c|c|c|c|c|c|c|c|c|c|c|c|c|}
\hline SDO & 0.11 & $\begin{array}{c}0.05- \\
0.16\end{array}$ & $<0.001$ & 0.11 & $\begin{array}{c}0.05- \\
0.17\end{array}$ & $<0.001$ & 0.10 & $\begin{array}{c}0.04- \\
0.16\end{array}$ & 0.001 & 0.09 & $\begin{array}{c}0.03- \\
0.15\end{array}$ & 0.003 \\
\hline CMQ & 0.20 & $\begin{array}{c}0.14- \\
0.26\end{array}$ & $<0.001$ & 0.20 & $\begin{array}{c}0.14- \\
0.26\end{array}$ & $<0.001$ & 0.14 & $\begin{array}{c}0.08- \\
0.20\end{array}$ & $<0.001$ & 0.14 & $\begin{array}{c}0.08- \\
0.20\end{array}$ & $<0.001$ \\
\hline CRT & -0.03 & $\begin{array}{c}-0.08- \\
0.03\end{array}$ & 0.373 & -0.03 & $\begin{array}{c}-0.08- \\
0.03\end{array}$ & 0.372 & -0.10 & $\begin{array}{l}-0.16- \\
-0.04\end{array}$ & 0.001 & -0.10 & $\begin{array}{l}-0.16- \\
-0.04\end{array}$ & 0.001 \\
\hline RWA*Threat & 0.04 & $\begin{array}{c}0.00- \\
0.08\end{array}$ & 0.048 & & & & 0.06 & $\begin{array}{c}0.02- \\
0.10\end{array}$ & 0.003 & & & \\
\hline SDO*Threat & & & & 0.03 & $\begin{array}{c}-0.02- \\
0.07\end{array}$ & 0.230 & & & & -0.03 & $\begin{array}{c}-0.07- \\
0.01\end{array}$ & 0.168 \\
\hline ICC & 0.39 & & & 0.39 & & & 0.53 & & & 0.53 & & \\
\hline \multirow[t]{2}{*}{$\mathrm{N}$} & $355_{\mathrm{ID}}$ & & & $355_{\mathrm{ID}}$ & & & $355_{\mathrm{ID}}$ & & & $355_{\mathrm{ID}}$ & & \\
\hline & \multicolumn{3}{|l|}{50 Stimuli } & \multicolumn{3}{|l|}{$50_{\text {Stimuli }}$} & \multicolumn{3}{|l|}{$50_{\text {Stimuli }}$} & \multicolumn{3}{|l|}{$50_{\text {Stimuli }}$} \\
\hline Observations & \multicolumn{3}{|c|}{3550} & \multicolumn{3}{|l|}{3550} & \multicolumn{3}{|l|}{3550} & \multicolumn{3}{|l|}{3550} \\
\hline $\begin{array}{l}\text { Marginal } \mathrm{R}^{2} / \\
\text { Conditional } \\
\mathrm{R}^{2}\end{array}$ & \multicolumn{3}{|c|}{0.091 / 0.444} & \multicolumn{3}{|c|}{$0.088 / 0.443$} & \multicolumn{3}{|c|}{$0.070 / 0.567$} & \multicolumn{3}{|c|}{$0.063 / 0.564$} \\
\hline
\end{tabular}

Table 3: Mixed-effects regression models predicting belief in and sharing of election-related misinformation among Republicans. All predictors and dependent variables are z-scores except threat, which is centered within stimuli then standardized.

\section{Non-Preregistered Analyses}

Exploratory analyses revealed potential concerns regarding the preregistered decision to "reverse code" belief and sharing responses to the seven true headlines in the stimuli set, in particular sharing intentions. We ran random-intercept only models to examine the variance explained by participants versus stimuli. For raw belief responses, the ratio of the random intercept standard deviation for participant/stimuli was 2.08 , suggesting $\sim 2 \mathrm{x}$ as much variance was explained by individual difference than difference between stimuli. Conversely, for sharing intentions the ratio was 7.78. In plotting the raw sharing values we found that the base rates of willingness to share were low $($ Mean $=2.56, S D=1.77$, Median $=2)$. In practical terms, many participants responded that they were "moderately unlikely" (the value corresponding to 2) to share both election-related misinformation and true headlines, and as such it may be ill-advised to "reverse code" responses for true headlines from 2 to 5 . Because of these concerns, we reran all the models reported above in Tables 2 and 3 with belief and sharing intention responses only for the 43 false stimuli (we removed all responses to the seven true headlines). We found that practically all results held. The only results that changed was that SDO was no longer a significant predictor of sharing intentions after controlling for party identification strength $(B=$ $0.05, p=0.157)$, although SDO still predicted sharing intention in the baseline model and in the threat model. As such, we are confident that our primary results conducted using the preregistered analyses were not driven by responses to the seven true headlines.

In an effort to inform theoretical questions related to the underlying mechanisms driving the observer associations between SDO/RWA and belief in/sharing of election-related 


\section{HIERARCHY-ENHANCING MISINFO}

misinformation, we ran models examining each scale's subscales and their associations with belief in and willingness to share election-related misinformation. Specifically, SDO's Dominance and Anti-Egalitarianism subscales (Ho et al., 2015), and RWA's Aggression, Traditionalism, and Submission subscales (Duckitt et al., 2010). Table 4 below displays the results. No concerns over multicollinearity arose across all models (highest VIF $=1.72$ ). The SDO-Dominance subscale was the only scale which was consistently associated with belief and sharing intention above party identification strength. SDO-Dominance consists of agreement with items such as "Some groups of people are simply inferior to other groups," "Groups at the bottom are just as deserving as groups at the top (reverse coded)," and "Some groups of people must be kept in their place."

Table 4

\begin{tabular}{|c|c|c|c|c|c|c|c|c|c|c|c|c|}
\hline \multirow[b]{2}{*}{ Predictors } & \multicolumn{3}{|c|}{$\begin{array}{c}\text { Belief in Election } \\
\text { Misinfo }\end{array}$} & \multicolumn{3}{|c|}{$\begin{array}{c}\text { Belief in Election } \\
\text { Misinfo }\end{array}$} & \multicolumn{3}{|c|}{$\begin{array}{c}\text { Sharing Election } \\
\text { Misinfo }\end{array}$} & \multicolumn{3}{|c|}{$\begin{array}{l}\text { Sharing Election } \\
\text { Misinfo }\end{array}$} \\
\hline & $\begin{array}{l}\text { Std. } \\
\text { Beta }\end{array}$ & $95 \% C I$ & $p$ & $\begin{array}{l}\text { Std. } \\
\text { Beta }\end{array}$ & $95 \% C I$ & $p$ & $\begin{array}{l}\text { Std. } \\
\text { Beta }\end{array}$ & $95 \% C I$ & $p$ & $\begin{array}{l}\text { Std. } \\
\text { Beta }\end{array}$ & $95 \% C I$ & $p$ \\
\hline (Intercept) & -0.00 & $\begin{array}{c}-0.09- \\
0.09\end{array}$ & 0.994 & -0.00 & $\begin{array}{c}-0.09- \\
0.09\end{array}$ & 0.994 & -0.00 & $\begin{array}{l}-0.13- \\
0.13\end{array}$ & 0.972 & -0.00 & $\begin{array}{l}-0.13- \\
0.13\end{array}$ & 0.972 \\
\hline $\begin{array}{l}\text { RWA } \\
\text { (Aggression) }\end{array}$ & 0.06 & $\begin{array}{c}-0.01- \\
0.14\end{array}$ & 0.110 & 0.05 & $\begin{array}{c}-0.02- \\
0.12\end{array}$ & 0.192 & -0.01 & $\begin{array}{c}-0.08- \\
0.07\end{array}$ & 0.840 & -0.02 & $\begin{array}{c}-0.10- \\
0.05\end{array}$ & 0.522 \\
\hline $\begin{array}{l}\text { RWA } \\
\text { (Traditionalism) }\end{array}$ & 0.11 & $\begin{array}{c}0.04- \\
0.18\end{array}$ & 0.003 & 0.05 & $\begin{array}{c}-0.02- \\
0.12\end{array}$ & 0.154 & 0.11 & $\begin{array}{c}0.04- \\
0.18\end{array}$ & 0.002 & 0.04 & $\begin{array}{c}-0.03- \\
0.11\end{array}$ & 0.219 \\
\hline $\begin{array}{l}\text { RWA } \\
\text { (Submission) }\end{array}$ & -0.02 & $\begin{array}{c}-0.10- \\
0.06\end{array}$ & 0.660 & -0.00 & $\begin{array}{c}-0.08- \\
0.07\end{array}$ & 0.903 & -0.02 & $\begin{array}{c}-0.09- \\
0.06\end{array}$ & 0.688 & -0.00 & $\begin{array}{c}-0.08- \\
0.07\end{array}$ & 0.993 \\
\hline $\begin{array}{l}\text { SDO } \\
\text { (Anti-Egal) }\end{array}$ & 0.02 & $\begin{array}{c}-0.07- \\
0.10\end{array}$ & 0.695 & 0.01 & $\begin{array}{c}-0.07- \\
0.09\end{array}$ & 0.859 & -0.07 & $\begin{array}{c}-0.16- \\
0.01\end{array}$ & 0.085 & -0.08 & $\begin{array}{c}-0.16- \\
-0.01\end{array}$ & 0.037 \\
\hline $\begin{array}{l}\text { SDO } \\
\text { (Dominance) }\end{array}$ & 0.13 & $\begin{array}{c}0.05- \\
0.21\end{array}$ & 0.002 & 0.11 & $\begin{array}{c}0.03- \\
0.19\end{array}$ & 0.008 & 0.20 & $\begin{array}{c}0.11- \\
0.28\end{array}$ & $<0.001$ & 0.17 & $\begin{array}{c}0.09- \\
0.25\end{array}$ & $<0.001$ \\
\hline Party ID Str & & & & 0.17 & $\begin{array}{c}0.10- \\
0.23\end{array}$ & $<0.001$ & & & & 0.21 & $\begin{array}{c}0.14- \\
0.27\end{array}$ & $<0.001$ \\
\hline ICC & 0.38 & & & 0.36 & & & 0.49 & & & 0.48 & & \\
\hline $\mathrm{N}$ & $\begin{array}{l}355_{\text {ID }} \\
50_{\text {Stimu }}\end{array}$ & & & $\begin{array}{l}355_{\text {ID }} \\
50_{\text {Stimuli }}\end{array}$ & & & $\begin{array}{l}355_{\text {ID }} \\
50_{\text {Stimuli }}\end{array}$ & & & $\begin{array}{l}355_{\mathrm{ID}} \\
50_{\text {Stimuli }}\end{array}$ & & \\
\hline Observations & 3550 & & & 3550 & & & 3550 & & & 3550 & & \\
\hline $\begin{array}{l}\text { Marginal } \mathrm{R}^{2} / \\
\text { Conditional } \mathrm{R}^{2}\end{array}$ & 0.042 & / 0.403 & & $0.066 /$ & 0.404 & & $0.037 /$ & 0.513 & & $0.072 /$ & 0.514 & \\
\hline
\end{tabular}

Table 4: Mixed-effects regression models predicting belief in and sharing of election-related misinformation among Republicans. All predictors and dependent variables are z-scores.

We then attempted to construct models which were the most parsimonious and generalizable for understanding belief in and willingness to share election-related misinformation. Based on the preregistered and non-preregistered findings, we removed RWA, replaced SDO with SDO-Dominance, removed CRT from the belief model, and entered 
perceived threat as a non-interactive variable, all with demographic controls (age, gender, ethnicity, and education). Table 5 displays those models.

Table 5

\begin{tabular}{|c|c|c|c|c|c|c|}
\hline \multirow[b]{2}{*}{ Predictors } & \multicolumn{3}{|c|}{ Belief in Election Misinfo } & \multicolumn{3}{|c|}{ Sharing of Election Misinfo } \\
\hline & Std. Beta & $95 \% C I$ & $p$ & Std. Beta & $95 \% C I$ & $p$ \\
\hline (Intercept) & -0.29 & $-0.66-0.08$ & 0.131 & -0.24 & $-0.69-0.21$ & 0.291 \\
\hline SDO-Dominance & 0.10 & $0.05-0.16$ & $<0.001$ & 0.13 & $0.06-0.20$ & $<0.001$ \\
\hline CMQ & 0.19 & $0.13-0.24$ & $<0.001$ & 0.10 & $0.03-0.17$ & 0.005 \\
\hline PID Str & 0.12 & $0.06-0.19$ & $<0.001$ & 0.16 & $0.09-0.23$ & $<0.001$ \\
\hline Perceived Threat & 0.06 & $0.01-0.11$ & 0.014 & 0.14 & $0.10-0.17$ & $<0.001$ \\
\hline CRT & & & & -0.08 & $-0.15--0.01$ & 0.021 \\
\hline ICC & \multicolumn{3}{|l|}{0.38} & \multicolumn{3}{|l|}{0.64} \\
\hline \multirow[t]{2}{*}{$\mathrm{N}$} & \multicolumn{3}{|l|}{$355_{\mathrm{ID}}$} & \multicolumn{3}{|l|}{$355_{\mathrm{ID}}$} \\
\hline & \multicolumn{3}{|l|}{$50_{\text {Stimuli }}$} & \multicolumn{3}{|l|}{$43_{\text {Stimuli }}$} \\
\hline Observations & \multicolumn{3}{|l|}{3550} & \multicolumn{3}{|l|}{3047} \\
\hline Demographic Controls & \multicolumn{3}{|l|}{ Yes } & \multicolumn{3}{|l|}{ Yes } \\
\hline True Headlines Included & \multicolumn{3}{|l|}{ Yes } & \multicolumn{3}{|l|}{ No } \\
\hline Marginal $\mathrm{R}^{2}$ / Conditional $\mathrm{R}^{2}$ & \multicolumn{3}{|l|}{$0.116 / 0.457$} & \multicolumn{3}{|c|}{$0.196 / 0.707$} \\
\hline
\end{tabular}

Table 5: Mixed-effects regression models predicting belief in and sharing of election-related misinformation among Republicans. All predictors and dependent variables are z-scores except threat, which is centered within stimuli then standardized.

\section{General Discussion}

We found that social dominance orientation was consistently positively associated with belief in and sharing of election-related misinformation among Republicans, as was conspiracy mentality and party identification strength. Contrary to our hypotheses, right-wing authoritarianism was not consistently associated with belief in or sharing of election-related misinformation, especially after controlling for party identification strength. Cognitive reflectiveness was only positively associated with sharing, but not belief. These findings support our theorizing that misinformation which challenges the legitimacy of fair and free elections is believed and shared in part because it is aligned with motives to maintain unequal social hierarchies which may be attenuated through democratic processes.

In examining the dynamics of support for election-related misinformation we found that perceived threat positively interacted with RWA, but not SDO, in its association with belief and sharing, suggesting that concerns about symbolic threats to the "American way of life" are not contributing to the relationship between SDO and election-related misinformation. Rather, we found that the SDO-Dominance subscale was driving the SDO association rather than the SDO-Anti Egalitarianism subscale. This suggests that overt motives for group-based dominance and oppression of low status groups are implicated in Republicans' rejection of the 2020 US 


\section{HIERARCHY-ENHANCING MISINFO}

election results, rather than motives to maintain social hierarchies through more subtle anti-egalitarian ideologies (see Ho et al., 2015 for a detailed discussion of these two subscales of SDO).

This work, while preliminary, makes two primary contributions, the first of which relates to generalizability of past work. Recent work has found that domain-general cognitive processes, such as cognitive reflectiveness, better explain support for belief and sharing of misinformation than ideologically motivated processes, and that the sharing of misinformation tends to be greater than the belief in it (for an overview see Pennycook \& Rand, 2021). Yet we observed the opposite pattern for election-related misinformation, as ideological processes and belief in and sharing of election-related misinformation were consistently associated, and we found that belief was higher than the willingness to share such misinformation (50.4\% vs. $32.5 \%)$. This divergence in findings is likely due to past work focusing on genericized and relatively innocuous political misinformation. These misinformation stimuli sets rarely contain information explicitly endorsed by conservatives elites, and the base rates of belief in this misinformation is low, for example Study 1 of Pennycook et al. (2021) found that such false headlines were rated as accurate $10-20 \%$ of the time. This suggests that past work may not generalize well to the context of hierarchy-enhancing misinformation.

More broadly, these findings help illuminate how misinformation can serve a functional role in reinforcing oppressive social hierarchies. Past work has highlighted how external State actors spread misinformation to increase local intergroup conflict, for example Russia's actions during the 2016 US election (Linvill et al., 2019; Linvill \& Warren, 2020), or that the spreading of misinformation may be driven by profit motives (Chen et al., 2019). Hierarchy-enhancing misinformation is conversely more likely to be spread by internal political actors, especially members of high status groups, with the intent of inducing feelings of group-based threat which drive engagement in group-based domination and hierarchy maintenance (Duckitt \& Sibley, 2010). Our findings parallel the observed relation between SDO (but not RWA) and climate change denialism (Häkkinen \& Akrami, 2014), another domain where misinformation potentially serves to reinforce existing social hierarchies. Expanding upon the theoretical framework of hierarchy-enhancing misinformation will help us understand how best to combat misinformation which reinforces oppression and erodes democratic norms.

Several limitations of the current work highlight fruitful avenues of future research. First, these findings, while generalizable across a large set of US 2020 election-related misinformation, are correlational. Experimental work is needed to support the causal link between hierarchy-enhancing motives and belief in election-related misinformation. Second, this work focuses exclusively on self-identified Republicans. While we argue this choice is ecologically appropriate given the phenomenon, caution is necessary when making inferences to other populations, for example conservative Independents, the general population, or left-wing authoritarians (Costello et al., 2021) in the United States or elsewhere who may oppose fair and free elections for reasons other than hierarchy-enhancing motives. 


\section{HIERARCHY-ENHANCING MISINFO}

To conclude, we call upon fellow social scientists studying misinformation to refocus their efforts toward understanding and combating election-related misinformation. Belief that the 2020 U.S. election was "stolen" from Donald Trump has not waned among Republican voters or officials either since the votes were counted or the January 6th attack on the US Capital. We argue this is best understood as a continuation of years of democratic backsliding in the US (Mickey et al., 2017; Waldner \& Lust, 2018) rather than an isolated incident of motivated reasoning or conformity to elite rhetoric. For example, many states are so extremely gerrymandered that experts agree some states no longer meet the definition of a democracy (Norris, 2020; Norris \& Gromping, 2019). These political realities should not be divorced from the sudden emergence of election-related misinformation following the 2020 US election. Understanding such misinformation in the context of other hierarchy-enhancing social and psychological processes is necessary if social scientists are to be effective at both attenuating misinformation and promoting democracy. 


\section{Acknowledgements}

We thank members of the Disinformation Working Group and the Sidanius Lab for their helpful feedback on study design and interpretation of the results.

\section{Funding}

This research was funded by Clemson University's Media Forensics Hub. Funds were used to compensate participants.

\section{Ethics}

This research was approved by Clemson University’s Institutional Review Board. All participants provided informed consent prior to participation, and no deception was utilized in this study.

\section{Contributions}

JL proposed the original research question. JL and VP contributed equally to all aspects of theorizing, hypothesis development, study design and the preregistion, and drafting of the manuscript. JL facilitated the analyses and prepared the open materials on the OSF. 


\section{References}

Agiesta, J., \& Edwards-Levy, A. (2021, September 15). CNN Poll: Most Americans feel democracy is under attack in the US. CNN.

https://www.cnn.com/2021/09/15/politics/cnn-poll-most-americans-democracy-under-atta ck/index.html

Barr, D. J., Levy, R., Scheepers, C., \& Tily, H. J. (2013). Random effects structure for confirmatory hypothesis testing: Keep it maximal. Journal of Memory and Language, 68(3), 255-278. https://doi.org/10.1016/j.jml.2012.11.001

Bates, D., Maechler, M., Bolker, B., \& Walker, S. (2015). Fitting linear mixed-effects models using lme4. Journal of Statistical Software, 67(1), 1-48. https://doi.org/10.18637/jss.v067.i01

Bratt, C., Sidanius, J., \& Sheehy-Skeffington, J. (2016). Shaping the development of prejudice: Latent growth modeling of the influence of social dominance orientation on outgroup affect in youth. Personality and Social Psychology Bulletin. https://doi.org/10.1177/0146167216666267

Bruder, M., Haffke, P., Neave, N., Nouripanah, N., \& Imhoff, R. (2013). Measuring individual differences in generic beliefs in conspiracy theories across cultures: Conspiracy mentality questionnaire. Frontiers in Psychology, 4. https://doi.org/10.3389/fpsyg.2013.00225

Chen, T., Liu, W., Fang, Q., Guo, J., \& Du, D.-Z. (2019). Minimizing misinformation profit in social networks. IEEE Transactions on Computational Social Systems, 6(6), 1206-1218. https://doi.org/10.1109/TCSS.2019.2944120

Choma, B. L., \& Hanoch, Y. (2017). Cognitive ability and authoritarianism: Understanding support for Trump and Clinton. Personality and Individual Differences, 106, 287-291. https://doi.org/10.1016/j.paid.2016.10.054

Costello, T. H., Bowes, S., Stevens, S. T., Waldman, I., Tasimi, A., \& Lilienfeld, S. O. (2021). Clarifying the structure and nature of left-wing authoritarianism. Journal of Personality \& Social Psychology. https://doi.org/10.31234/osf.io/3nprq

Craig, M. A., \& Richeson, J. A. (2014a). More diverse yet less tolerant? How the increasingly diverse racial landscape affects white americans' racial attitudes. Personality \& Social Psychology Bulletin, 40(6), 750-761. https://doi.org/10.1177/0146167214524993

Craig, M. A., \& Richeson, J. A. (2014b). On the precipice of a "majority-minority" America: Perceived status threat from the racial demographic shift affects White Americans' political ideology. Psychological Science, 25(6), 1189-1197.

Duckitt, J., Bizumic, B., Krauss, S. W., \& Heled, E. (2010). A tripartite approach to right-wing authoritarianism: The authoritarianism-conservatism-traditionalism model. Political Psychology, 31(5), 685-715. https://doi.org/10.1111/j.1467-9221.2010.00781.x

Duckitt, J., \& Sibley, C. G. (2010). Personality, ideology, prejudice, and politics: A dual-process motivational model. Journal of Personality, 78(6), 1861-1894. https://doi.org/10.1111/j.1467-6494.2010.00672.x

Enders, C. K., \& Tofighi, D. (2007). Centering predictor variables in cross-sectional multilevel 


\section{HIERARCHY-ENHANCING MISINFO}

models: A new look at an old issue. Psychological Methods, 12(2), 121-138. https://doi.org/10.1037/1082-989X.12.2.121

Federico, C. M., Weber, C. R., Ergun, D., \& Hunt, C. (2013). Mapping the connections between politics and morality: The multiple sociopolitical orientations involved in moral intuition. Political Psychology, 34(4), 589-610. https://doi.org/10.1111/pops.12006

Frederick, S. (2005). Cognitive reflection and decision making. Journal of Economic Perspectives, 19(4), 25-42. https://doi.org/10.1257/089533005775196732

Garrett, R. K., \& Bond, R. M. (2021). Conservatives' susceptibility to political misperceptions. Science Advances, 7(23), eabf1234. https://doi.org/10.1126/sciadv.abf1234

Häkkinen, K., \& Akrami, N. (2014). Ideology and climate change denial. Personality and Individual Differences, 70, 62-65. https://doi.org/10.1016/j.paid.2014.06.030

Halali, E., Dorfman, A., Jun, S., \& Halevy, N. (2018). More for us or more for me? Social Dominance as parochial egoism. Social Psychological and Personality Science, 9(2), 254-262. https://doi.org/10.1177/1948550617732819

Ho, A. K., Sidanius, J., Kteily, N., Sheehy-Skeffington, J., Pratto, F., Henkel, K. E., Foels, R., \& Stewart, A. L. (2015). The nature of social dominance orientation: Theorizing and measuring preferences for intergroup inequality using the new $\mathrm{SDO}_{7}$ scale. Journal of Personality and Social Psychology, 109(6), 1003-1028. https://doi.org/10.1037/pspi0000033

Ho, A. K., Sidanius, J., Pratto, F., Levin, S., Thomsen, L., Kteily, N., \& Sheehy-Skeffington, J. (2012). Social dominance orientation: Revisiting the structure and function of a variable predicting social and political attitudes. Personality and Social Psychology Bulletin, $38(5), 583-606$.

Jost, J. T., van der Linden, S., Panagopoulos, C., \& Hardin, C. D. (2018). Ideological asymmetries in conformity, desire for shared reality, and the spread of misinformation. Current Opinion in Psychology, 23, 77-83. https://doi.org/10.1016/j.copsyc.2018.01.003

Judd, C. M., Westfall, J., \& Kenny, D. A. (2017). Experiments with more than one random factor: Designs, analytic models, and statistical power. Annual Review of Psychology, 68(1), 601-625. https://doi.org/10.1146/annurev-psych-122414-033702

Kraus, M. W., Rucker, J. M., \& Richeson, J. A. (2017). Americans misperceive racial economic equality. Proceedings of the National Academy of Sciences, 114(39), 10324-10331. https://doi.org/10.1073/pnas.1707719114

Linvill, D. L., Boatwright, B. C., Grant, W. J., \& Warren, P. L. (2019). “THE RUSSIANS ARE HACKING MY BRAIN!” investigating Russia's internet research agency twitter tactics during the 2016 United States presidential campaign. Computers in Human Behavior, 99, 292-300. https://doi.org/10.1016/j.chb.2019.05.027

Linvill, D. L., \& Warren, P. L. (2020). Troll Factories: Manufacturing Specialized

Disinformation on Twitter. Political Communication, 37(4), 447-467. https://doi.org/10.1080/10584609.2020.1718257

Maertens, R., Anseel, F., \& van der Linden, S. (2020). Combatting climate change 


\section{HIERARCHY-ENHANCING MISINFO}

misinformation: Evidence for longevity of inoculation and consensus messaging effects. Journal of Environmental Psychology, 70, 101455. https://doi.org/10.1016/j.jenvp.2020.101455

Maertens, R., Roozenbeek, J., Basol, M., \& van der Linden, S. (2020). Long-term effectiveness of inoculation against misinformation: Three longitudinal experiments. Journal of Experimental Psychology: Applied. https://doi.org/10.1037/xap0000315

Mickey, R., Levitsky, S., \& Way, L. (2017). Is America still safe for democracy: Why the United States is in danger of backsliding. Foreign Affairs, 96(3), 20-29.

Norris, P. (2020). Electoral integrity in the 2020 U.S. elections (p. 23). Election Integrity Project. https://www.electoralintegrityproject.com/peius2020

Norris, P., \& Gromping, M. (2019). Electoral integrity worldwide (p. 37). Election Integrity Project. https://www.electoralintegrityproject.com/the-year-in-elections-2019

Nyhan, B., Porter, E., Reifler, J., \& Wood, T. J. (2020). Taking fact-checks literally but not seriously? The effects of journalistic fact-checking on factual beliefs and candidate favorability. Political Behavior, 42(3), 939-960. https://doi.org/10.1007/s11109-019-09528-x

Onyeador, I. N., Daumeyer, N. M., Rucker, J. M., Duker, A., Kraus, M. W., \& Richeson, J. A. (2021). Disrupting beliefs in racial progress: Reminders of persistent racism alter perceptions of past, but not current, racial rconomic equality. Personality and Social Psychology Bulletin, 47(5), 753-765. https://doi.org/10.1177/0146167220942625

Palan, S., \& Schitter, C. (2018). Prolific.ac-A subject pool for online experiments. Journal of Behavioral and Experimental Finance, 17, 22-27. https://doi.org/10.1016/j.jbef.2017.12.004

Pennycook, G., Binnendyk, J., Newton, C., \& Rand, D. G. (2021). A practical guide to doing behavioral research on fake news and misinformation. Collabra: Psychology, 7(1), 25293. https://doi.org/10.1525/collabra.25293

Pennycook, G., Epstein, Z., Mosleh, M., Arechar, A. A., Eckles, D., \& Rand, D. G. (2021). Shifting attention to accuracy can reduce misinformation online. Nature. https://doi.org/10.1038/s41586-021-03344-2

Pennycook, G., \& Rand, D. (2021a). The psychology of fake news. Trends in Cognitive Sciences, 15. https://doi.org/10.1016/j.tics.2021.02.007

Pennycook, G., \& Rand, D. G. (2019). Lazy, not biased: Susceptibility to partisan fake news is better explained by lack of reasoning than by motivated reasoning. Cognition, 188, 39-50. https://doi.org/10.1016/j.cognition.2018.06.011

Pennycook, G., \& Rand, D. G. (2021b). Research note: Examining false beliefs about voter fraud in the wake of the 2020 Presidential Election. Harvard Kennedy School Misinformation Review. https://doi.org/10.37016/mr-2020-51

Roozenbeek, J., \& van der Linden, S. (2019). The fake news game: Actively inoculating against the risk of misinformation. Journal of Risk Research, 22(5), 570-580. https://doi.org/10.1080/13669877.2018.1443491 
Ross, R. M., Rand, D. G., \& Pennycook, G. (2021). Beyond "fake news": Analytic thinking and the detection of false and hyperpartisan news headlines. Judgment and Decision Making, 16(2), 484-504. https://doi.org/10.31234/osf.io/cgsx6

Sidanius, J., Cotterill, S., Sheehy-Skeffington, J., Kteily, N., \& Carvacho, H. (2016). Social dominance theory: Explorations in the psychology of oppression. In The Cambridge Handbook of the Psychology of Prejudice (pp. 1-58).

Son Hing, L. S., Bobocel, D. R., Zanna, M. P., \& McBride, M. V. (2007). Authoritarian dynamics and unethical decision making: High social dominance orientation leaders and high right-wing authoritarianism followers. Journal of Personality and Social Psychology, 92(1), 67-81. https://doi.org/10.1037/0022-3514.92.1.67

Thomson, K. S., \& Oppenheimer, D. M. (2016). Investigating an alternate form of the cognitive reflection test. Judgment and Decision Making, 11(1), 15.

Waldner, D., \& Lust, E. (2018). Unwelcome change: Coming to terms with democratic backsliding. Annual Review of Political Science, 21(1), 93-113. https://doi.org/10.1146/annurev-polisci-050517-114628

Walter, N., Cohen, J., Holbert, R. L., \& Morag, Y. (2020). Fact-checking: A meta-analysis of what works and for whom. Political Communication, 37(3), 350-375. https://doi.org/10.1080/10584609.2019.1668894

Womick, J., Rothmund, T., Azevedo, F., King, L. A., \& Jost, J. T. (2019). Group-based dominance and authoritarian aggression predict support for Donald Trump in the 2016 U.S. presidential election. Social Psychological and Personality Science, 10(5), 643-652. https://doi.org/10.1177/1948550618778290 